\title{
Modeling the Effects of Contraceptives as a Control Strategy in Human Population Dynamics
}

\author{
Kirui Wesley ${ }^{1}$, Rotich Titus ${ }^{2}$ \\ ${ }^{1}$ Department of Mathematics and Actuarial Science, South Eastern Kenya University, Kitui, Kenya \\ ${ }^{2}$ Department of Mathematics and Physics, Moi University, Eldoret, Kenya
}

Email Address:

kirwes@yahoo.com (K. Wesley), tisesko@yahoo.com (R. Titus)

\section{To cite this article:}

Kirui Wesley, Rotich Titus. Modeling the Effects of Contraceptives as a Control Strategy in Human Population Dynamics. Mathematical Modelling and Applications. Vol. 4, No. 2, 2019, pp. 22-35. doi: 10.11648/j.mma.20190402.12

Received: June 15, 2019; Accepted: July 22, 2019; Published: August 16, 2019

\begin{abstract}
The population size of every country or government is very important in planning on effective service delivery. The cost of conducting population census yearly is of great significance to the country's budget and many countries conduct population census once in a decade. This makes planning and provision of services to be based on mere approximation. Provision of free maternity services, estimation of national hospital insurance fund premium for medical care, and provision of retirement benefits, payment of allowances to the aged require accurate demographic statistics. In this study, population dynamics is described using a stochastic model, where population is put into distinct and disjoint age classes: Juvenile, subAdult, Adult, Resting-Adult, Senior Citizens and the Aged. These structures are assigned intra and inter group transmission rates which form the elements of transmission matrix and presented in form of a Leslie model. The model was modified to allow stochastic variation of transition parameters which is affected by demographic and environmental factors, specifically the effect of contraceptives to control population. It was found that intermittent implementation of control strategy at $50 \%$ and $70 \%$ efficacy yields a steady population growth rate of $\lambda=1.39$ and a steady population distribution of $P=(23 \%, 10 \%, 23 \%$, $18 \%, 23 \%, 20 \%, 6 \%)^{T}$.
\end{abstract}

Keywords: Population Control, Employment Ratio, Replacement Rate, Economic Ratio, Force Dependency Ratio, Leslie Model, Dependency Ratio, Demographic Parameters

\section{Introduction}

The study of human population involves the analysis of the dynamics of numbers and composition in a given region over a given span of time and the factors that influence those changes. Some population dynamics arise due to changes in birth rate, death rates, immigration and emigration, distribution, gender, aging and population structure.

Mathematical modeling and analysis of population dynamics is known as demography. It is the core business of every government to provide services to its citizens in terms of security, health education, employment, just to mention but a few. In order to do this effectively, there is need for demographic data and possibly a projection for better, planning and distribution of available resources [17]. This information is obtained by studying the previous changes in connected variables like mortality, fertility and migration. Appropriate mathematical analytic and simulation methods are then used to draw conclusions and make predictions of future human population. Nowadays mathematical modeling of population dynamics is a central topic in theoretical biology and modern biology. We use models in order to extract the trends from complex processes to permit comparison among systems, to facilitate analysis of causes of processes acting on the systems and to make prediction about the future [6].

The study of human Population dynamics is an interdisciplinary area of study where scholars from demography, epidemiology, sociology, anthropology study population in order to explains other related factors such as fertility rate, life expectancy, impact on economic, social and environmental systems which include quality of natural resources like biodiversity, air, water, land, energy supplies, food security and climate change.

Every government requires accurate information on its population in order to put realistic plans and management of 
its government in terms of provision of quality services and products to her citizens. In most cases, the knowledge of future population is required for the purpose of planning and budgeting for the citizens' provision of services.

Due to large amount of money required to conduct population census, most governments conduct census once after every 10 years. In between, the government roughly estimates the size of its population and thus planning using wrong data. This is such a long time that planning and budgeting will not reflect the desired effectiveness due to lack of accurate population size, and thus there is need to operate on exact population size of its citizens every year.

Most regions in the world are experiencing rapid demographic change because of huge expansion in human population [3]. With very high increase in human population, Kenyan government invests huge money in running public health campaigns, family planning units, sensitization on use of contraceptives in an attempt to control the population growth. These campaigns have not been successful due to lack of knowledge in parameters that determine the population growth. Currently, the government of Kenya is battling with providing free education, free medical service, free maternity service and free income to the senior citizens, widows and orphans. This support is effective if well planned, and the planning heavily depends on the availability of accurate data.

Demographers deal with the collection, presentation and analysis of data relating to the basic life-cycle events. They also examine the compositions of population by sex, age, ethnicity, occupation, marital status and religion. This brings the need to use mathematical models to determine the threshold values of population control strategies and predict the population at any desired time. This can be determined using sex and age structured stochastic mathematical models [15]. Leslie as a demographer used a variety of counts to measure fertility, mortality, migration and other population dynamics. Leslie modeling approach involves the use of current trends in mortality, fertility, migration and immigration.

\subsection{Historical Development of Mathematical Modeling in Population}

History of mathematical modeling in population studies began in 1202, with Fibonacci in the book Liber Abaci, which introduced the sequence, later named after him, to describe the growth of rabbit population [22]. This made demography, the only part of life sciences which used mathematics in the $20^{\text {th }}$ century [3]. Halley, mostly known as the astronomer published in 1693 two articles of life annuities based on mortality tables for the city of Breslau [4]. This work highly influenced the development of life insurance. In 1798, Malthus [16] claimed that the human population grows exponentially with constant growth rate. This was later improved by Verhulst [26] who proposed a bounded, self limiting population model defined by the equation commonly known as logistic equation. This models is more realistic because the abundance of a species is dependent on the carrying capacity of the limited resources [23]. It was observed that the reproduction and survival of individuals decrease for smaller populations and it can lead to the extinction of population depending on the availability of resources. This phenomenon was later called Allee effect [1]. Ricker [20] invented a discrete model which was used in fishery stocks. This model got recognition and was applied throughout in the study of population dynamics. The model was of the form

$$
P_{t+1}=P_{t} \exp \left\{r\left(1-\frac{P_{t}}{M}\right)\right\}
$$

Note in the equation above that the density dependence in this model becomes stronger at higher densities, due to the exponential function relating density to the carrying capacity. A more generalized discrete model proposed by Hansel, [11] is defined as;

$$
P_{t+1}=\frac{\lambda P_{t}}{\left(1+a P_{t}\right)^{b}}
$$

Many population studies have used deterministic models which assume constant growth rate, death rate and other parameters. Ideally, these parameters are often affected by both environmental and demographic factors for example occurrence of a catastrophe, government policy on family planning and many other factors. These variations due to demographic and environmental conditions are captured in a stochastic model. The population projections made using deterministic model is therefore unrealistic and it is for this reason that this study aimed at analyzing the population dynamics using stochastic model.

\subsection{Leslie Model}

Studies on dynamical processes involving stochastic growth rate have been explored by researchers and applied to population dynamics of various species and their interactions. Leslie models in $[14,15]$ are similar to exponential equation $P(t)=P_{0} e^{\lambda t}$. Williamson [27] studied a discrete model using Leslie matrix with age structures such that the first row contained elements representing fertility and the entries below the diagonal represent the death rates of each structure. This was further developed by Usher, [25] in the study of forest trees. This model was applied in insect population with the distinct metamorphic stages [13]. The Leslie model is therefore given by recurrence relation,

$$
P_{k+1}=L P_{k} ; k \in \mathbb{Z}^{+}
$$

where $k$ is the time interval or season long enough for change in population to happen. The closed form solution of Equation (1.5) is given by;

$$
P_{k}=L^{k} P_{0}
$$

Usher, [24] and Goodman, [8] together showed that Leslie basic matrix is split into the matrices, $F$ for birth rate and $S$ for survival rates, and the full Leslie matrix $L=F+S$. The matrices $F$ and $S$ are of the form; 


$$
F=\left(\begin{array}{cccc}
\mathrm{F}_{1} & \mathrm{~F}_{2} & \ldots & \mathrm{F}_{\mathrm{m}} \\
0 & 0 & \ldots & 0 \\
\vdots & \vdots & \ddots & \vdots \\
0 & 0 & 0 & 0
\end{array}\right), S=\left(\begin{array}{cccc}
0 & 0 & \ldots & 0 \\
\mathrm{~s}_{1} & 0 & \ldots & 0 \\
\vdots & \vdots & \ddots & \vdots \\
0 & 0 & \mathrm{~s}_{\mathrm{m}-1} & 0
\end{array}\right) \text { and } L=\left(\begin{array}{cccc}
\mathrm{F}_{1} & \mathrm{~F}_{2} & \ldots & \mathrm{F}_{\mathrm{m}} \\
\mathrm{s}_{1} & 0 & \ldots & 0 \\
\vdots & \vdots & \ddots & \vdots \\
0 & 0 & \mathrm{~s}_{\mathrm{m}-1} & 0
\end{array}\right)
$$

Where $s_{i} ; i=1,2,3, \ldots m-1$ denotes survival rates to the next class or group and $F_{i} ; i=1,2,3, \ldots, m$ denotes the birth rates for each class or cluster with similar characteristics. This means that the Leslie matrix $L$ may not be constant throughout the simulation. This is because demographic factors like fertility, death rate, survival rate are different for each cluster due to modification by environmental and catastrophic factors and thus vary the values of the matrices $S$ and $F$.

There are many reasons for describing population using structured models. These models provide for the inclusion of different demographic characteristics of individuals of different ages and gender. The choice of the age structured model for the human population is to capture exactly the specific population ages that are responsible for the production of the new generation. Using the entire population may be unrealistic because the young and the old do not contribute to the growth rate.

Using Leslie matrix, the growth rate factor, can be modified to accommodate the effects of demographic and environmental stochasticity. In this case, the study informs the way forward as part of the long term plans for controlling the population.

From the research findings, the government can be able to plan well for the creation and allocation of the limited resources to the precisely known number of citizens. This can best be done if the population is known. Since plans are made in advance, it is necessary that the best tool to project the population in the future time is available so that as budget is made the government is fully certain of the population. It shows the composition of a population in terms of sex, age and the regional distribution. It also provides information on trends and levels of mortality and fertility. It assists the government in knowing whether there are adequate economic and social resources to maintain high standards of living [6].

Also, other researchers will benefit from the study by using the derived results and concepts to apply in other population studies. The techniques of analysis presented here will be beneficial to apply to any country or in a smaller way to any county for easy planning.

In addition, population prediction is of benefit to the politicians who believe in the tyranny of numbers while requesting for votes. The population prediction techniques when used per region or community can help determine the number of expected voters that are legible as registered voters for the purpose of election.

The interaction of animals in an environment leads eventually to an equilibrium point where the different species coexist [21]. This equilibrium is a point which depends on the environmental ability to support the interacting species. This also applies to human being but the carrying capacity is always improved by human activities [28]. Population of animals and their distribution depends on the ecological conditions of the environment. Likewise, we find that the density of human settlement depends on the environmental conditions of the locations where they have settled.

\subsection{Population and Reproduction}

In this study, the general objective was to formulate a mathematical model to describe the population dynamics and estimate the threshold values of parameters determining population dynamics. In particular, the control strategy of mitigating population explosion is evaluated to determine the desirable level of efficacy. Several models can be used to achieve this but the most subtle and realistic model is Leslie matrix. This is because Leslie matrix method allows for the subdivision of population into age structures basing on their demographic characteristics. Here, the fertility and the mortality rates of different strata created can be modified using population control strategies. This is motivated by the fact that young people of ages between 0 and 14 are not mature enough to reproduce. And therefore their fertility rate is almost zero. The ages between 15 and 24 are school going ages and despite being productive, their reproduction is restricted due to schooling, and therefore their fertility is low. The next age group of between 25 and 44 comprises the married, whose core business is to bring up children. This will therefore have the highest fertility rate. After this age group, most women are in their menopause stage and their reproduction rates very low. After 65 years, fertility is zero.

\subsection{Population and Economic Productivity}

Productivity of individuals in various employment careers depends on age. Most people in the labour force bracket and actively employed in self or formal employment are between ages 25 and 65 . These are the populations who support the other age groups. The maintenance of the old and young brings the biggest burden to every government and any plans to balance the various age groups for self-sustenance is welcomed. The quality of life in a country depends on this people. The ability of the citizens to economically support itself depends on the ratio of the employed to unemployed. The parameter measuring the ratio of the employed and the rest of the population is a significant parameter in planning. The ratio of these groups and the rest determines the economic strength of the country. Denote the working class as $W c$ and the young and the old by $Y$ and $O l$ respectively. Then the economic ratio is defined as;

$$
\text { EconRatio }=\mathrm{e}\left(\frac{W c}{Y+O l}\right):=R_{e}
$$

where $e$ is the threshold parameter accounting for the proportion of labour force (self or formal sector) people of 
age between 25 and 65, here called the working group. This value depends on the average rate of expenditure an individual needs and the amount of earnings they get. It is desirable that $R_{e}>1$. The interpretation is that, if $R_{e}>1$, the employed citizens are able to support the unemployed, and if $R_{e}<1$, the country needs to plan to support the unemployed in terms of medical, home settlement, free education just to mention but a few.

The different dynamics in each stratum is best analyzed using stochastic models. Leslie model is the most suitable strategy, in analyzing reproductive health control strategies in order to achieve desirable economic interdependence.

\section{Literature Review}

The increase of aged people in a population due to declines in fertility and mortality rates is a characteristic of developed countries. In recent years, the issue of population ageing has received renewed attention in many countries, especially those in the more developed regions, owing to the continuance of fertility below the replacement level and ongoing trends towards lower mortality. The phenomenon of population ageing leads to population leveling followed by a decline in some future time.

Population control strategies used to obtain a desired structure are reviewed in this section. These strategies include age reversal and growing of young population. The most important parameters to play with are; fertility rate, mortality rate and immigration policies. The desired population structure must yield positive economic implications [19].

Increasing fertility rate slows down the ageing process of a population. In order to make a young population to age, there is need to control this fertility rate. The use of contraceptives plays a key role in any attempt to achieve an economically viable population structure [2].

When population is studied using age structured, parameters determining the movement from one structure to another are evaluated using survival rate, death rate and birth rate which are vital functions of age [12]. Varying the vital rates with time transforms Leslie model making it a function of [5].

\subsection{Population in Kenya}

Large population has negative impacts on the environment and on the available resources. However, large population is not a challenge to all countries. Population control is a concern to developing nations due to un-supporting economies. This problem of population is noticeable in SubSaharan Africa. In particular, Kenya's population has more than quadrupled since 1963, growing from more than $8.1 \mathrm{~m}$ to more than $43 \mathrm{~m}$ people in 2010 . Today, Kenya has the sixth highest population in sub-Saharan Africa, behind Tanzania, South Africa, the Democratic Republic of the Congo, Ethiopia and Nigeria. Life expectancy has risen from 48 in 1963 to 60 in 2011, above the 56-year average for SubSaharan Africa.

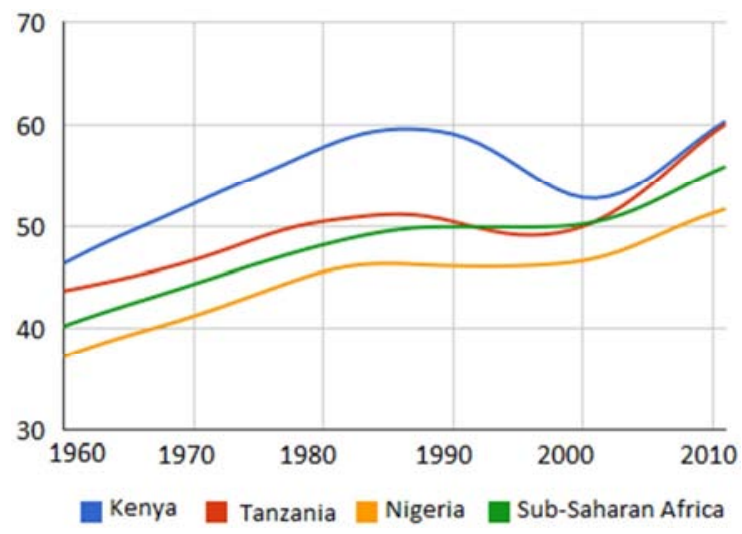

Figure 1. Life Expectancy Population Trends from 1960 - 2010. Source: [30].

According to the World Banks data, the fertility rate in Kenya was 7.9 in 1960 and 4.3 in 2014. Also, the death rates were 20 for 1960 and 8 in 2014. This indicates that the population in 2014 is declining since the difference in fertility rates is negative.

The following is a summary of the metadata from World Bank on Kenya Population.

Table 1. Kenyan Demographic MetaData. Source: [31].

\begin{tabular}{|c|c|c|c|c|c|}
\hline Characteristic & Gender & Age & Value & & \\
\hline Year & & & 1960 & 2014 & 2015 \\
\hline \multirow{2}{*}{ Life Expectancy } & Female & & 48 & 63 & \\
\hline & Male & & 44 & 60 & \\
\hline Birth Rate & & & 7.9 & 4.3 & \\
\hline Death Rate & & & 20 & 8 & \\
\hline Life Expectancy at Birth & & & 46 & 62 & \\
\hline Infant Mortality Rate & $0-1$ & & 66 & & 36 \\
\hline \multirow{2}{*}{ Child mortality rate } & Female & $<5$ & 102 & 49 & 45 \\
\hline & Male & & & & 49 \\
\hline \multirow{2}{*}{ Adult Mortality Rate } & Female & & & & 59 \\
\hline & Male & & & & 240 \\
\hline
\end{tabular}

http://databank.worldbank.org/data/reports. Accessed on 28th August 2016 at 2.30pm

The following scenario is experienced in Kenya's population. The age structure, and gender is summarized in the table below. 
Table 2. Population Distribution in Kenya as at 2014. (CIA World Factbook).

\begin{tabular}{|c|c|c|c|c|}
\hline Age Group & Label & Percentage & Male & Female \\
\hline $0-14$ years & Juvenile & $42.1 \%$ & 9,494983 & $9,435,795$ \\
\hline $15-24$ years & Sub-Adult & $18.7 \%$ & $4,197,382$ & $4,202,399$ \\
\hline $25-54$ years & Adults & $32.8 \%$ & $7,458,665$ & $7,302,34$ \\
\hline $55-64$ years & Resting Adults & $3.7 \%$ & 751,296 & 910,23 \\
\hline 65 years and over & Senior Citizens & $2.8 \%$ & 548,431 & 708,048 \\
\hline
\end{tabular}

The information on this table is summarized on population pyramid of Kenya's population in 2014 is depicted in Figure 2 below.

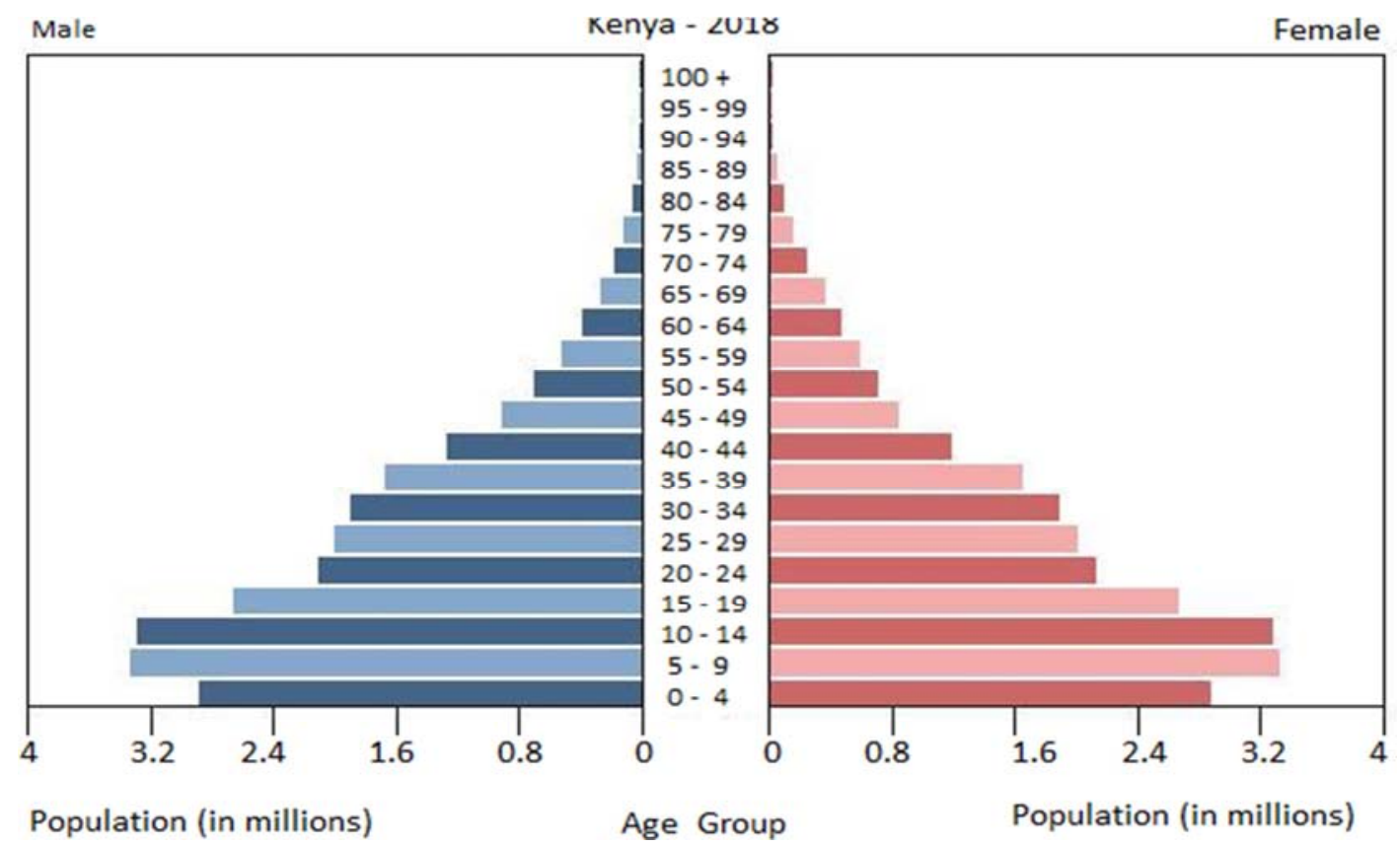

Figure 2. Kenya Population Pyramid. Source: CIA World Factbook-(last updated on 30th June, 2015).

Age pyramids are equally important in policy formulation. By comparing the population sizes of different ages, issues like social security, medical care, education can easily be discussed because population per age can be directly interpreted. Consider the population of USA and India based on census dating back from 1966 [7].

\subsection{Contraceptives and Population Control}

Among many other strategies used in population control according to http://listcrux.com, (2016), include; Delayed Marriage, improved medical Facilities; Legislative guidelines; providing incentives; Increased education and awareness of negative impact of overpopulation; Women Empowerment; Poverty eradication [10] and access to contraceptives.

Among the population control strategies mentioned, the more pronounced and easily implemented, monitored and controlled is the use of contraceptives. Contraceptive prevalence rate is the percentage of actively reproductive women in ages (15-49 years) using family planning methods. When estimating contraceptive prevalence an assumption is made that women between the ages of 15-49 are sexually active and therefore are also exposed to the risk of pregnancy [9].

Contraceptive use varies substantially among world nations. The 2010 World Population Data Sheet shows that contraceptive prevalence rate vary widely from an average of $75 \%$ in America to $29 \%$ in Africa. A number of factors are responsible for the low contraceptive prevalence rates among sexually active women in Africa. Reasons for noncontraceptive use range from difficulties in accessing fertility centers, fertility-related infections, traditional beliefs, and ignorance on birth control methods. In addition poor coordination of reproductive health programs is one additional factor contributing to low contraceptive use in Africa.

The use of contraceptives in all the regions in the world generally corresponds with fertility patterns [9]. Fertility is the ratio of live births in an area to the population of that area. Contraceptive use is directly related to fertility rate, as depicted in Table 3 below. Clearly, there is a direct correlation between contraceptive prevalence rate and the Total Fertility rate. 
Table 3. Population Distribution and Other Demographic Parameters.

\begin{tabular}{lllllll}
\hline Region /Country & Population (Millions) & Birth rate & Death rate & Growth rate & Fertility rate & Percentage use of Contraceptives \\
\hline World & 6.892 & 20 & 8 & 1.2 & 2.5 & 62 \\
Developed & 1.237 & 11 & 10 & 0.2 & 1.7 & 71 \\
Less developed & 5.656 & 22 & 8 & 1.4 & 2.7 & 60 \\
Africa & 1030 & 37 & 13 & 2.4 & 4.7 & 29 \\
Kenya & 40.0 & 37 & 10 & 2.7 & 4.6 & 46 \\
\hline
\end{tabular}

Source: Population Reference Bureau. World Population Data Sheet. 2010.

Fertility rates have fallen in some of the world regions have dropped from 5.0 around the 1950 s to 2.5 in 2009. But for some regions, including Africa, the rate remains quite high [10].

In this study, contraceptives, which obviously reduces the fertility rate is incorporated into the elements of Leslie matrix, and the modified matrix used to simulate the effect of contraceptive use.

\section{Formulation of Governing Equations}

The study of population dynamics is an area that has been of much interest to researchers, politicians and the government officials for the purpose of population management, planning and provision of services. Of much interest is the ability to project the future population of a certain country, cluster or an ecosystem. This section gives the analytic methods used to achieve desired objectives of using contraceptives to control population growth. The section is subdivided into three; development of the model equations, Leslie matrix and analysis of the model.

\subsection{Stochastic Model}

Many mathematical models use deterministic models to approximate the future population. These methods are not realistic due to the fact that environmental and stochastic factors affect the reproductive rate (growth rate and/or survival rate). In order to incorporate these factors, this study will use a stochastic population model and subdivide the entire population into structures basing on their distinct survival and reproductive rates. As discussed earlier, the environmental and demographic stochasticities are captured in a stochastic population model equivalent to equation (6) defined by;

$$
P_{t+1}=P_{t}+\lambda P_{t} ; P(0)=P_{0}
$$

By iteration for $t=0$ to $t=n$, the solution of (3. 5) is obtained to be:

$$
P_{n}=(1+\lambda)^{n} P_{0}
$$

The advantage of the solution given in equation (7) is that the growth factor $\lambda$ can be modified depending on the demographic or environmental factors that affects the growth rate. In an occurrence of a catastrophe, the growth factor $\lambda$ will be reduced, say to $\lambda_{c} \leq \lambda$. In such a time say at time $m$, the population at an earlier time $m-1$ is described by:

$$
P_{m-1}=(1+\lambda)^{m-1} P_{0}
$$

and at the time of the occurrence of the catastrophe $m$, the population is determined as:

$$
P_{m}=\left(1+\lambda_{c}\right)(1+\lambda)^{m-1} P_{0}
$$

If the catastrophe occurs $k$ times $k \leq n$ then the entire population after $n$ time seasons will be described as

$$
P_{n}=\left(1+\lambda_{c}\right)^{k}(1+\lambda)^{n-k} P_{0}
$$

\subsection{Leslie Model}

Despite the beauty of a stochastic model in capturing many dynamics, it is still insufficient because of the assumption that the entire population is homogeneously productive and contributes equally to the population growth. Children under 10 years old and the old above 50 years contribute very little if not none to the growth. Similarly, the mortality rates of people are not uniform for infants as it is for adults and the aged. This calls for the need to study the population in distinct age-structures with age specific survival rates and age specific birth rates. This is handled using Leslie model in equation (3), with Leslie matrix defined in equation (5) and the solution in equation (4).

\subsection{Effects of Demographic Control Strategies on Leslie Matrix}

The population has grown by $30 \%$ between 1990 and 2010 [19]. Adverse effects related to this has become a challenge to many countries, especially in China, India and Africa.

Many a times, a nation will use several population control strategies to manage the growth rate. Some of these strategies include; family planning, policy on maximum children, support of a fixed number of children to discourage many children among many other ways [29]. The target group of the population control strategies is to the actively productive age group, usually between 18 years old and 35 years old. This will affect the birth rate of the class of individuals in the category and also the eventual growth rate as a whole.

Population control strategies are aimed at planning for the provision of quality service to the people in an equitable manner. Unknown population size will interfere with the planning of provision of services to the citizens.

Generally, family planning strategies aims at reducing or increasing the population growth rate. This means from Equation (8), the adjusted growth rate parameter $\lambda_{c}$ accounts for the effect of family planning strategy. Similarly, from 
Equation (4), the Leslie matrix population projection equation with adjusted fertility and survival rates, say $L_{c}$, we obtain the Equation:

$$
P_{n}=L_{c}^{k} L^{n-k} P_{0}
$$

$$
L_{c}=\left(\begin{array}{cccccc}
F_{1 c} & F_{2 c} & F_{3 c} & F_{4 c} & \cdots & F_{m c} \\
\mathrm{~s}_{1 c} & 0 & 0 & 0 & \cdots & 0 \\
0 & S_{2 c} & 0 & 0 & \cdots & 0 \\
0 & 0 & S_{3 c} & 0 & \cdots & 0 \\
0 & 0 & 0 & \ddots & \cdots & 0 \\
0 & 0 & 0 & 0 & \mathrm{~s}_{(m-1) c} & 0
\end{array}\right)
$$

where, $F_{i c}, i=1,2, \ldots, m$ is the adjusted fertility rate and $\mathrm{s}_{i c}, i=1,2, \ldots, m-1$ is the adjusted survival rate after incorporating population control strategy.

When population intervention strategy is applied to $i^{\text {th }}$ age group, and since $F_{i}$ denotes the fertility rate of the $i^{\text {th }}$ age group, the fertility rate affected in the next generation will be

$$
\left(\begin{array}{c}
N_{0} \\
N_{1} \\
N_{2} \\
\vdots \\
N_{m}
\end{array}\right)=\left(\begin{array}{ccccc}
F_{1 c} & F_{2 c} & F_{3 c} & \ldots & F_{m c} \\
s_{1 c} & 0 & 0 & \ldots & 0 \\
0 & s_{2 c} & 0 & \ldots & 0 \\
\vdots & \vdots & \ddots & \ldots & 0 \\
0 & 0 & 0 & \mathrm{~s}_{(m-1) c} & 0
\end{array}\right)^{\mathrm{k}}\left(\begin{array}{ccccc}
F_{1} & F_{2} & F_{3} & \ldots & F_{m} \\
\mathrm{~s}_{1} & 0 & 0 & \ldots & 0 \\
0 & s_{2} & 0 & \ldots & 0 \\
\vdots & \vdots & \ddots & \ldots & 0 \\
0 & 0 & 0 & s_{(m-1)} & 0
\end{array}\right)^{\mathrm{n}-\mathrm{k}}\left(\begin{array}{c}
N_{0} \\
N_{1} \\
N_{2} \\
\vdots \\
N_{m}
\end{array}\right)
$$

where the subscript $c$ denotes the modified Leslie matrix accounting for contraceptives, and $m$ is the number of age structures subdividing the entire population while $k$ is the number of times contraceptives is used.

\subsection{Eigen Analysis of Leslie Matrix}

The Eigen analysis is the determination of the characteristic roots of the transition matrix $L$. The dominant eigenvalue of the matrix $L$ yields the population growth rate, with the right eigenvector as the stable population distribution and the left eigenvector as the unstable population distribution.

For every Leslie matrix $L$, there is a dominant eigenvalue, say $\lambda_{1}$ which is strictly greater than the other eigenvalues and this dominant eigenvalues determines the asymptotic properties of the population. The corresponding eigenvector $\tilde{v}_{1}$ is real and describes the dominant population distribution which is stable for each population class. This dominant eigenvalue determines the ultimate nature of the population, as to whether the population goes into extinction, explodes with growth, or if it exhibits a periodic behaviour. To understand why all these cases occur, the eigenvalues and eigenvectors of the Leslie matrix are investigated. For large values of time, the age distribution vector is a scalar multiple of the eigenvector associated with the largest eigenvalue of the Leslie matrix. If there is a single dominant eigenvalue $\lambda^{*}$ of the Leslie matrix, with associated normalized eigenvector $\tilde{v}^{*}$, then asymptotically the population grows like

$$
P_{n}=c^{*}\left(\lambda^{*}\right)^{n} \tilde{v}^{*}
$$

It follows that the population grows (if $\lambda^{*}>1$ ) or decays
$F_{i+1}$ and if the same effect is repeated every season, then after $n$ years/seasons, the fertility rates will have changed for all except the new born age group.

The projected population will therefore be derived from Equation (3.4) which is given in its expanded form as;

(if $\lambda^{*}<1$ ) much like Malthusian growth model with the exponential growth $e^{\lambda t}$ and the age classes having a distribution of $\tilde{v}^{*}$.

\subsection{Numerical Solutions}

The numerical scheme used in this study was the finite difference numerical scheme. The model equations will be simulated using Crank-Nicholson method. This method is known for its rate of convergence and high precision, [18]. The numerical results will be evaluated using initial parameter values obtained from Kenya Bureaus of Statistics. The results will therefore be used to validate the analytic results of the model. Results will be discussed if the comparison of model results tally with field data. Poor fit of the model will be calibrated and computation done again. When the results are satisfactory, conclusion and recommendation will be made.

\section{Data Analysis and Presentation}

In this section, the analytic results in the previous section are justified using numerical solutions. This is done using data of a given species to make the interpretations, projections and conclusions that mimics the reality. Simulation of these results is done using Runge-Kutta of order 4 and 5 numerical scheme in MATLAB.

\subsection{Fertility Rate}

Fertility rate as defined before indicates the number of offsprings of an individual at a certain age over a given time. Most women are reproductive between ages 15 and 45 years. 
The number of children born to woman within period of child bearing age or reproductive age is referred to as fertility. Fertility is a meaningful measure of demography. To estimate the number of children to woman is based on the meaningful of fertility. In fact, the problem related to population is not how excessively people being born but few. The two important things that one must know about births are the total fertility rate and the replacement rate. The data available for Kenya shows that adults are at a fertility rate value of 4.6 children per individual. Using the data in Table 1, Table 2 and Table 3, the Kenyan population is analyzed using Runge - Kutta numerical scheme in MATLAB for the purpose of projection.

\subsection{Analysis and Population Projection}

The structure of the population is subdivided into five age brackets as described in Table 1 , that is $0-14,14-$ $24,25-54,55-64$ and 65 and above. The respective mortality and fertility rates are given in Table 2 and Table 3. Under normal conditions where no population control strategy is employed, we formulate Leslie projection matrix using the data in Table 4 below.

Table 4. Demographic Parameters of Kenya in 2015 in Thousands (000).

\begin{tabular}{lllllll}
\hline Age & Female & Male & Total & Percentage & Fertility & Mortallity \\
\hline $0-14$ & 9436 & 9495 & 18931 & $42.1 \%$ & 0.045 \\
$15-24$ & 4202 & 4197 & 8399 & $18.7 \%$ & 0.059 \\
$25-54$ & 7303 & 7459 & 14762 & $32.8 \%$ & 4.6 \\
$55-64$ & 911 & 751 & 1662 & $3.7 \%$ & 4.6 \\
$65-$ above & 708 & 548 & 1256 & $2.8 \%$ & 0.5 & 0.24 \\
Total & 22451 & 22559 & 45010 & 1 & 0.36 & 0.5 \\
\hline
\end{tabular}

Basing on the above table, the Leslie Matrix $L$ and the initial population distribution $P$ for our model is given by;

$$
L=\left(\begin{array}{ccccc}
0 & 0.059 & 0.24 & 0.36 & 0.5 \\
0 & 0 & 0 & 0 & 0 \\
0 & 4.6 & 0 & 0 & 0 \\
0 & 0 & 4.6 & 0 & 0 \\
0 & 0 & 0 & 0.5 & 0
\end{array}\right) \text { and } P=\left(\begin{array}{c}
18,931,000 \\
8,399,000 \\
14,762,000 \\
1,662,000 \\
1,256,000
\end{array}\right)
$$

Using this matrix, the next population after one cycle or reproductive period is evaluated using the relation in equation (3). The following table shows the values generated in MATLAB using the data in Table 4.

\subsection{Model Numerical Simulation}

The following data was generated using Runge-Kutta numerical scheme and the data in Table 1, Table 2 and Table 3. Simulation results for the distribution of population and growth factor for various intervention strategies is given by;

Table 5. Simulation results of Population Projection in absence of Intervention (in Ten Thousands 00000).

\begin{tabular}{lllrrrrrrrrrrrr}
\hline Time Category & $\boldsymbol{t}=\mathbf{0}$ & $\mathbf{t}=\mathbf{1}$ & $\boldsymbol{t}=\mathbf{2}$ & $\boldsymbol{t}=\mathbf{3}$ & $\boldsymbol{t}=\mathbf{4}$ & $\boldsymbol{t}=\mathbf{5}$ & $\boldsymbol{t}=\mathbf{6}$ & $\boldsymbol{t}=\mathbf{7}$ & $\boldsymbol{t}=\mathbf{8}$ & $\boldsymbol{t}=\mathbf{9}$ & $\boldsymbol{t}=\mathbf{1 0}$ & $\boldsymbol{t}=\mathbf{1 1}$ & $\boldsymbol{t}=\mathbf{1 2}$ & $\boldsymbol{t}=\mathbf{1 3}$ \\
\hline Juvenile & 189 & 53.77 & 128.33 & 218.08 & 239.82 & 231.04 & 134.51 & 199.92 & 231.89 & 214.81 & 191.76 & 192.11 & 221.35 & 219.67 \\
Sub-Adults & 84 & 113.4 & 32.262 & 76.999 & 130.85 & 143.89 & 138.63 & 80.708 & 119.95 & 139.13 & 128.88 & 115.06 & 115.27 & 132.81 \\
Adults & 106 & 218.4 & 294.84 & 83.881 & 200.2 & 340.21 & 374.11 & 360.43 & 209.84 & 311.88 & 361.74 & 335.1 & 299.15 & 299.69 \\
Resting Adults & 42 & 190.8 & 393.12 & 530.71 & 150.99 & 360.36 & 612.37 & 673.4 & 648.77 & 377.71 & 561.38 & 651.14 & 603.18 & 538.47 \\
Senior Citizens & 17 & 50.4 & 228.96 & 471.74 & 636.85 & 181.18 & 432.43 & 734.85 & 808.08 & 778.53 & 453.25 & 673.66 & 781.37 & 723.81 \\
The Old & 13 & 6.8 & 20.16 & 91.584 & 188.7 & 254.74 & 72.473 & 172.97 & 293.94 & 323.23 & 311.41 & 181.3 & 269.46 & 312.55 \\
\hline
\end{tabular}

The Dominant eigenvalue $\lambda^{*}$ of matrix $L$ and the distribution is determined using the MATLAB code from the matrix below. Clearly, the dominant eigenvalue $\lambda^{*}=1.3964$ as indicated in the simulation results below.

$$
\sigma(L)=\left(\begin{array}{cccccc}
1.3964 & 0 & 0 & 0 & 0 & 0 \\
0 & 0.061057 & 0 & 0 & 0 & 0 \\
0 & 0 & 0.061057 & 0 & 0 & 0 \\
0 & 0 & 0 & -0.65745 & 0 & 0 \\
0 & 0 & 0 & 0 & -0.65745 & 0 \\
0 & 0 & 0 & 0 & 0 & -0.20358
\end{array}\right)
$$

The dominant eigenvalue and the corresponding eigenvector representing the population distribution in percentage are:

\begin{tabular}{|c|c|c|c|c|c|c|}
\hline & Juvenile & Sub-Adults & Adults & Resting Adults & Senior Citizens & The Old \\
\hline Stratum & $(0-14)$ & $(15-24)$ & $(25-44)$ & $(45-54)$ & $(55-64)$ & (Over 65) \\
\hline Distribution in \% & 0.22721 & 0.097629 & 0.18178 & 0.23432 & 0.20137 & 0.057684 \\
\hline
\end{tabular}

Table 6. Population population Distribution per age stata. 
In absence of any population control intervention strategy, the population in Kenya is projected in Figure 3 below to hit a value of 100 million in 2030. This means the government will not have difficulties supporting the population because from the analysis by age, the resting adults of ages $45-54$ are the majority followed by the Juvenile $0-14$ years old and then adults $25-44$. Most of these groups are active in employment and thus can support the young and the aged. which are the minority.

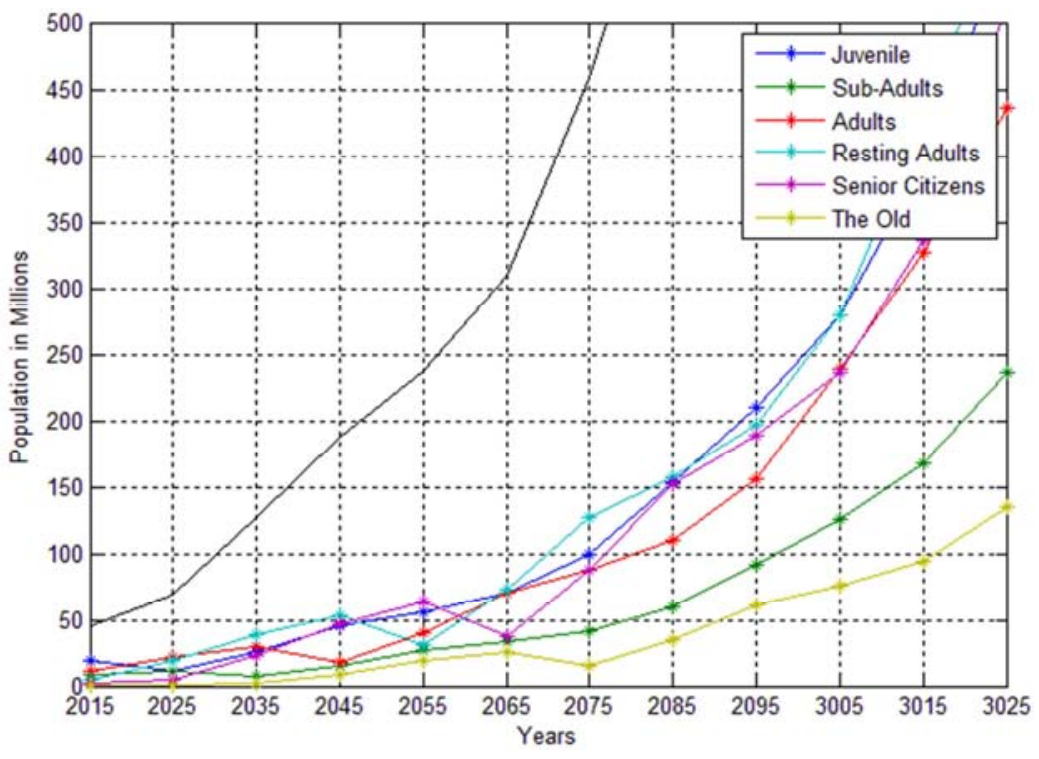

Figure 3. Population Dynamics with no Control Intervention Strategy.

Very large population has many disadvantages because of limited resources. There is need to control the population so that the limited resources can be able to support the population. Use of contraceptives to control population is seen in Table 2 to be at 0.0036 in Kenya, that is $46 \%$ of the people can access and use contraceptives. If a serious campaign is organized so that the fertility rate is reduced by $30 \%$, the total population can reduce significantly as shown in Figure 4 below. In Figure 4, the population control strategy is assumed to begin from 2020 and continue consistently for 35 years. This may mean that females are given some long lasting injection or other form of contraceptive lasting for 35 years. After the expiry of 35 year control, it is noticed that the population spirals back and continue growing exponentially. This means 2 generations of 15 years each will be affected.

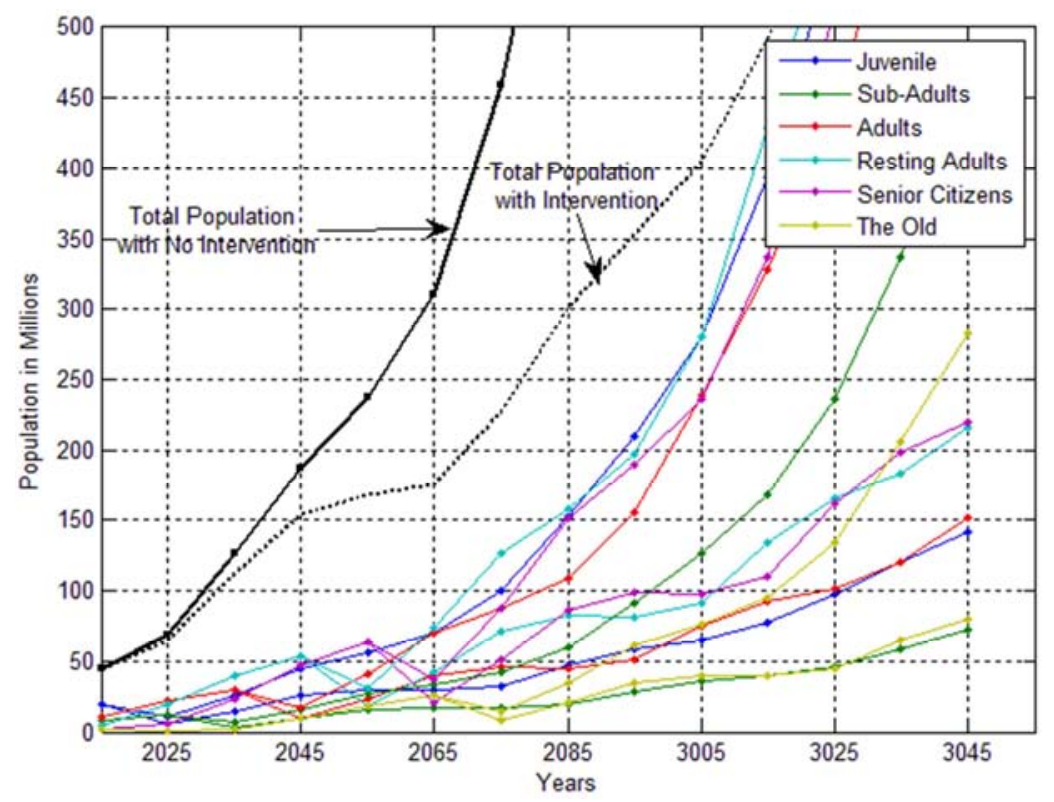

Figure 4. Intervention for 40 years at an efficacy of $40 \%$ from 2025 to 2065.

If the control strategy is administered once to last for 2 years, the affected population will reduce the fertility rate of 
the child bearing age only, but after five years, a new generation will have replaced the old and the population will continue growing. This is depicted in Figure 5 below. This means that a population control lasting only for two years has no substantial effect on the total population. There is need therefore to increase the campaign and increase the efficacy of the control strategies.

Figure 6 shows the effect of the total population at different levels of the population control efficacy. It is noted that desired population size is obtained if the control strategy is repeated periodically and at increasing efficacy.

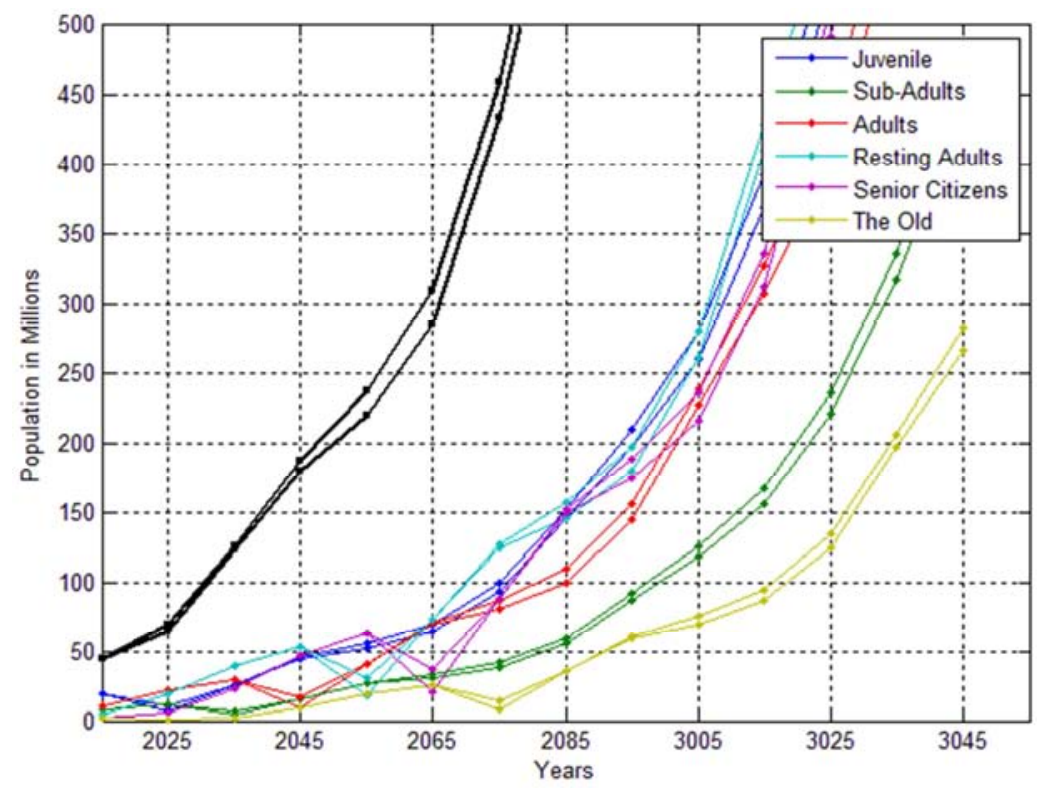

Figure 5. Implementation of Intervention control strategy once lasting for 2 years.

The intervention is applied after the first 5 years at $30 \%$, to last for 25 years, then increased to $50 \%$ to last for another 25 years, and lastly increased to $70 \%$ efficacy to last for 25 years. Clearly, by the year 3015, which is almost 100 years from now, the population will be at 110 million which is manageable, The highest attained by 3045 is $215,000,000$ people in Kenya.

To avoid the decline of population which is evidenced at years 2065 and 3015, the best strategy that can be applied in the control of population is by intermittent application of an efficacy of $50 \%$ and $70 \%$ interchanging at intervals of 5 years. The simulated results shows constant and gently growing population with no negative growth registered throughout the period. The intermittent control strategy is applied as follows, in 2020, a 50\% control is applied to all child bearing individuals, targeting women and men equally and then in 2055, the strategy is increased to $70 \%$. This should last up to 2065 when $50 \%$ strategy is implemented then $70 \%$ again in 2085 and $50 \%$ in 3015 .

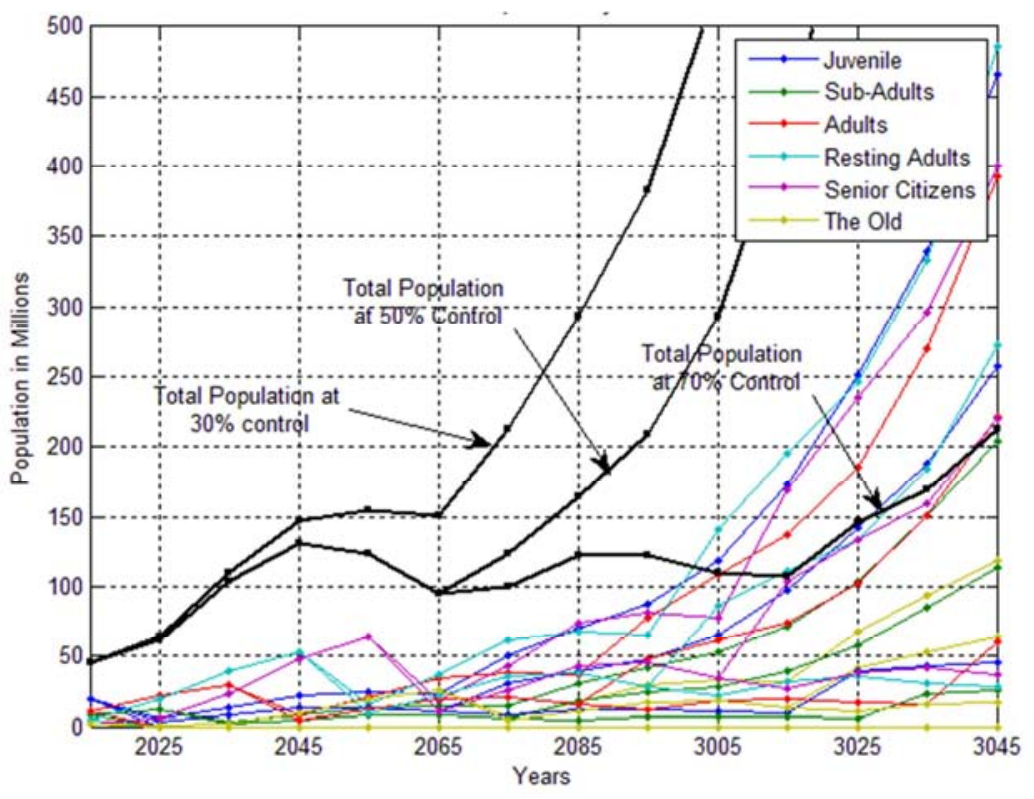

Figure 6. Intervention at 30\%, 50\%, 70\% starting in 2025 to last up to the year 3045 . 
This trend should be repeated continually so that the population will grow at a desired pace.

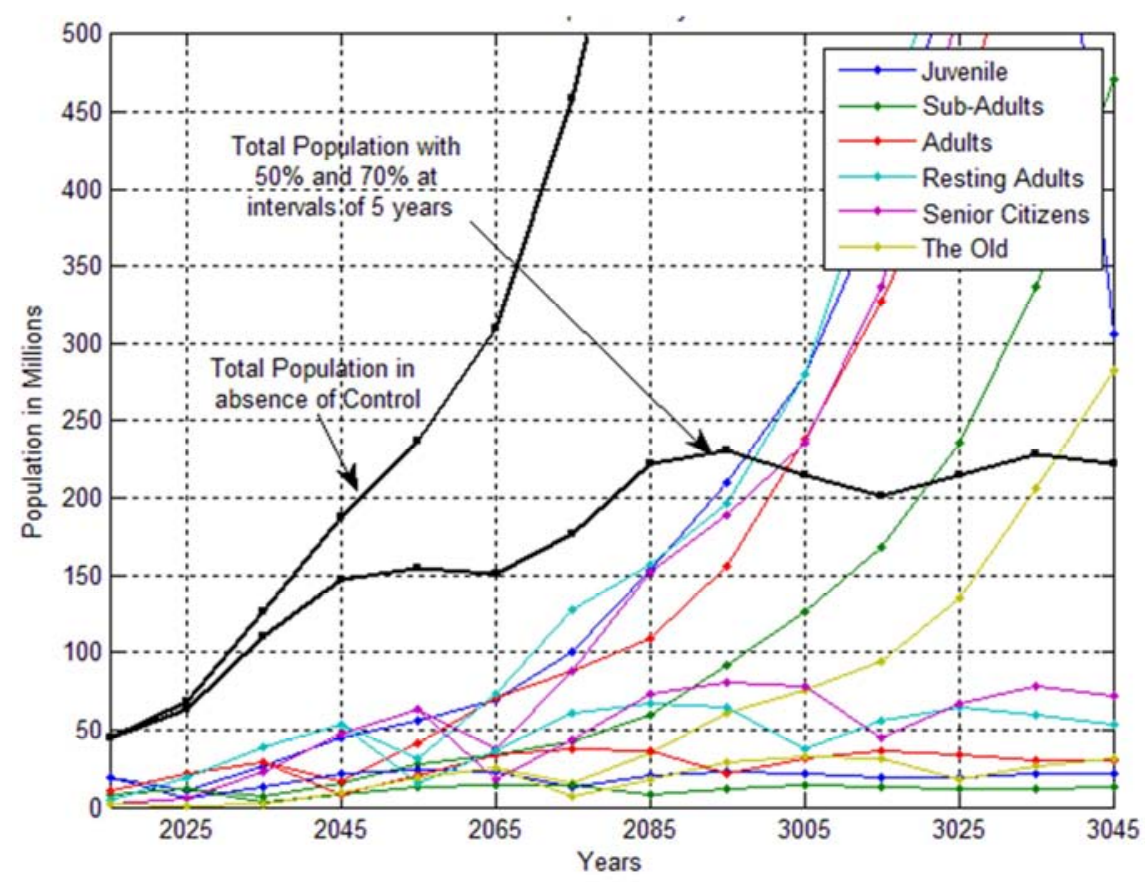

Figure 7. Intermittent control at $50 \%$ and $70 \%$ at intervals of 20 years interchangeably.

\subsection{Labor force Dependence Ratio}

This is the ratio of the work force between 25 and 65 years to the remaining population, defined by the equation

$$
R_{e}=\mathrm{e}\left(\frac{W c}{Y+O l}\right)
$$

where the parameters $e$ is the rate of employment among the workforce, $W c$ is the population of the work force, $Y$ is the population of the young and $O \mathrm{O}$ is the population of the old. It is here assumed that every employed individual earns enough income to maintain himself alone when $e=1$. The employee can maintain himself and $n$ others if $e=n+1$. The desire of everybody is to be able to support her fertility. The computation of the parameter $e$ is done using the data available in Table 4, the population distribution in 2015 is given by;

Table 6. Computation of Labor force dependence ratio.

\begin{tabular}{llll}
\hline Category & Age Group & $\begin{array}{l}\text { Population } \\
\text { (in thousands) }\end{array}$ & $\begin{array}{l}\text { Total per } \\
\text { category }\end{array}$ \\
\hline Young & $0-14$ & 18,931 & 27330 \\
& $15-24$ & 8399 & \\
Work force & $25-54$ & 14762 & 16424 \\
Old age & $55-64$ & 1662 & 1256 \\
& $65-$ above & 1256 & 45010 \\
\hline
\end{tabular}

Labor force dependence ratio is calculated as;

$$
R_{e}=\frac{e(16,424)}{27330+1256}=0.57454698 e
$$

With the assumption that all the work force are 100\% employed, that is $e=1$, the value of $R_{e}$ indicates that the workforce citizens are over stretched as the dependent group. It implies that at least, the income of every individual member of the workforce should earn enough to support at least two others, that is, $e \geq 2$. This is the condition necessary to make $R_{e}>1$.

Figure 8 below illustrates the level of dependence with and without population control strategies.

\subsection{Simulation of Labour Force Dependence Ratio}

The initial computation of the labour dependency ratio illustrated in section 4.5 can be repeated for every population distribution in subsequent years, including the periods where population control intervention is applied. From the simulation results using MATLAB, it is noticed that the value of the ratio $R_{e}$ oscillates as the population also oscillates, due to the use of contraceptives. In absence of control strategies, the dependent population increases at a higher rate than the workforce. When $e=1$, the value of $R_{e}$ approaches zero. Figure 8 shows the trend of the dependency ratio.

The following is simulation results of the dependent and the independent age groups. The dependent group is composed of ages $0-24$, and 65 - and above, while the independent group is composed of individuals falling between ages 25 and 64 . The independent group is what is here referred to as the labour force or the group of individuals who are actively employed or seeking employment. The next table shows dependency ratio for the rest of the time periods. 
Table 7. Value of Dependency Ratio for Different Values of Employment e.

\begin{tabular}{|c|c|c|c|c|}
\hline Values of $R_{e}$ Period (time) & $e=1$ & $e=1.5$ & $e=2$ & $e=3$ \\
\hline 1 & 1.7333 & 2.6 & 3.4667 & 5.2 \\
\hline 2 & 0.5002 & 0.7502 & 1.0003 & 1.5005 \\
\hline 3 & 0.3785 & 0.5678 & 0.7570 & 1.1355 \\
\hline 5 & 0.7550 & 1.1325 & 1.5101 & 2.2651 \\
\hline 6 & 0.7151 & 1.0726 & 1.4302 & 2.1453 \\
\hline 7 & 0.5181 & 0.7771 & 1.0361 & 1.5542 \\
\hline 9 & 0.6692 & 1.0039 & 1.3385 & 2.0077 \\
\hline 10 & 0.6375 & 0.9562 & 1.2750 & 1.9124 \\
\hline 11 & 0.6001 & 0.9002 & 1.2002 & 1.8003 \\
\hline 12 & 0.6052 & 0.9078 & 1.2104 & 1.8156 \\
\hline 13 & 0.6303 & 0.9455 & 1.2607 & 1.8910 \\
\hline 14 & 0.3625 & 0.5437 & 0.7250 & 1.0875 \\
\hline
\end{tabular}

Column three with $e=2$ marks the threshold value of employment rate necessary to sustain the population. The graphs for different values of dependency ratio $R_{e}$ at different values of employment rate $e$.

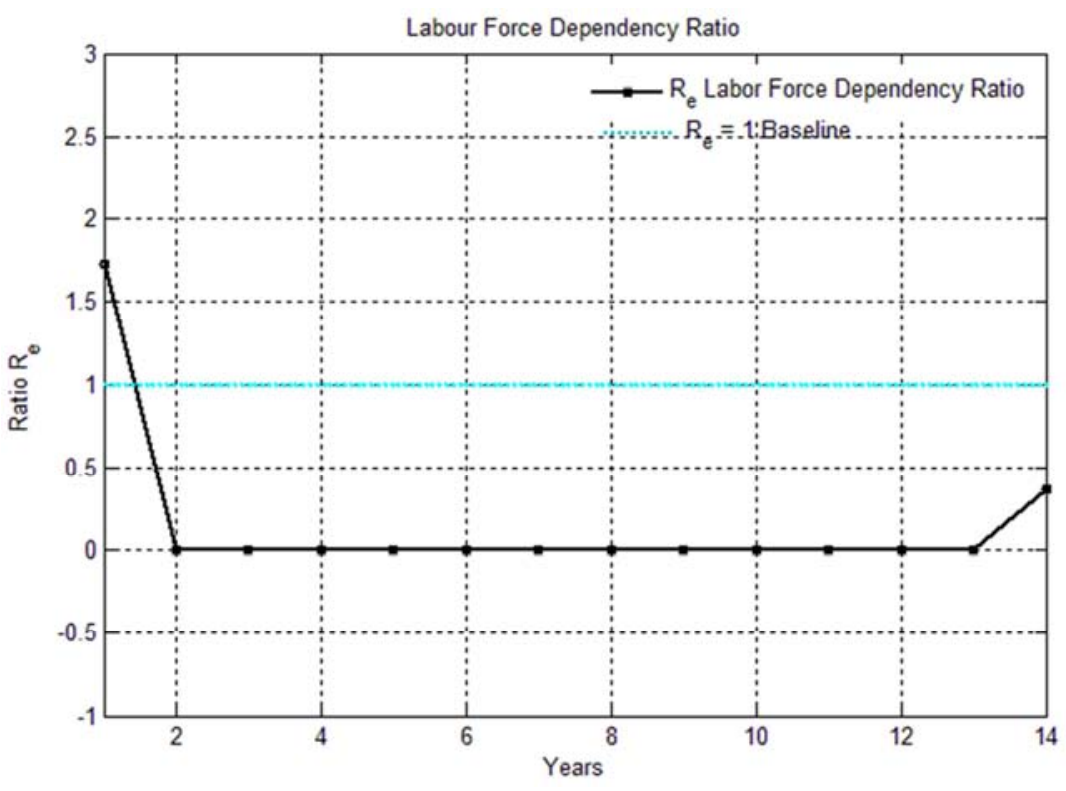

Figure 8. Dependency Ratio in absence Population Control Intervention Strategy.

The implementation of population control strategy, reduces the fertility rate of the control strategy, thus reducing the population size of the young, between $0-14$ years and meanwhile the ageing population is wiped out. This results into a majority population ranging between ages 25 and 65 , which is composed of the labour force. This therefore increases the dependency ratio to values closer to one, as illustrated in Figure 9 below.

Comparing with the population dynamics, the ratio is greater than one when the total population is low, and the ratio is much closer to zero, then the total population is highest. The oscillation is expected since the application of control strategies was intermittent.

The value of the dependency ratio can be improved if the government increases provision of free services to the old and the young.

This includes the provision of free education, free medical treatment to the young and to the old, and improvement of salary to the working, enough to support their families.

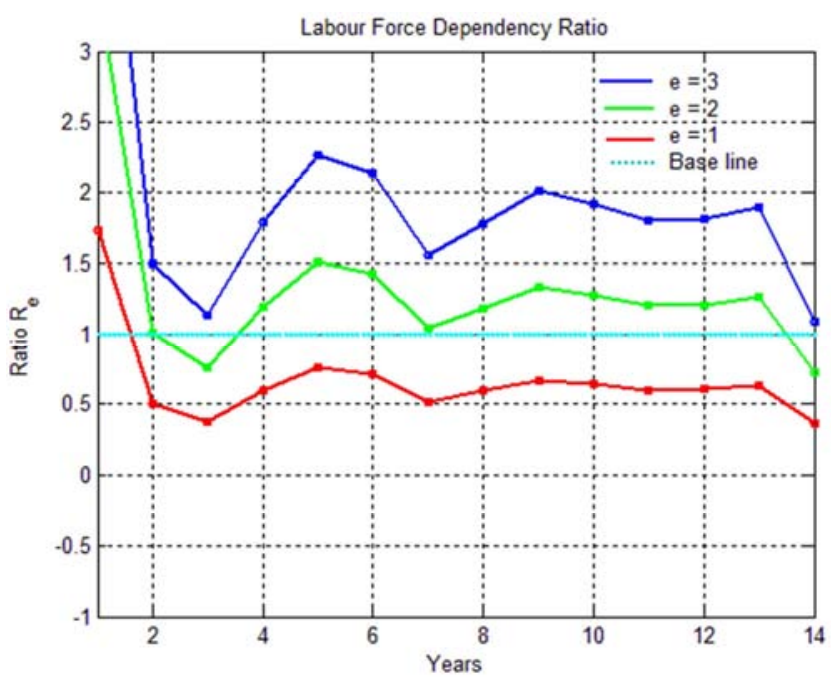

Figure 9. Labour Force Dependency Ratio in Presence of Control Strategies. 
The graphs above are uniformly translated up above the baseline $R_{e}=1$ by the employment rate factor $e$. The desired dependency rate should be above one. This is achieved if the employment rate $R_{e} \geq 2$.

\section{Summary, Conclusion and Recommendation}

In this chapter, a summary of the results is presented and respective conclusion and recommendation is made.

\subsection{Summary}

Using matrix method, Leslie matrix is used together with the secondary demographic data for Kenya. Simulation results shows that application of control strategies is very effective if it is implemented continually and to last long periods of time. The population recovers when the control strategy is suspended and the population will grow exponentially. It is found that $50 \%$ and $70 \%$ efficacy is desired. This means that individual fertility rate is reduced from the current 4.6 children per person to 2.3 at $50 \%$ and down to 1.5 at $70 \%$ control efficacy. The population will therefore increase at a slower pace so that it can be supported by the government. It clearly found here that the percentage difference between the initial population of 2015 and the final predicted population of 2025 shows a population increase of $10 \%$ in absence of population control intervention strategy, but will increase by $5 \%$ if the use of contraceptives is effectively implemented. The Labour force dependency ratio $R_{e}$ is found to be greater than one if the employment rate is greater or equal to 2 . This implies that the employed individuals should earn money enough to support two unemployed people. This can be made possibly by the government providing free medical cover and free education to the young (under 24) and the old (over 65 years).

\subsection{Conclusion}

Leslie matrix provides the most effective way of simulating population dynamics which includes the intervention or stochastic interference from the environment or demographic sources. The Leslie matrix $L$ and the modified Leslie matrix $L_{m}$ is used together to simulate the results when an intervention strategy is put in place. In this study, the population projection model yields a dominant growth rate of $\lambda=1.39$ in absence of intervention with a population distribution of $P=(23 \%, 10 \%, 23 \%, 18 \%, 23 \%$, $20 \%, 6 \%)^{\mathrm{T}}$ from the initial population distribution of $P=(42.1,18.7,33.8,3.7,2.8)$.

It is found that the intervention strategy of using contraceptives at $50 \%$ efficacy and $70 \%$ efficacy is very effective in controlling the population. The model used to simulate intermittent control strategy was $P_{n+1}=$ $L^{n-k-m} L_{c}^{k} L_{d}^{m} P_{n}$ where $k<n$ and $m<n$ accounts for the number of times the control strategy was applied at $50 \%$ and at $70 \%$ respectively, while $n$ is the entire period of experiment.
Under these conditions, the labour force dependency ratio $R_{e} \geq 1$ if the threshold value of employment rate $e \geq 2$. Provision of free support to the young and the old citizens by the government will lead to the achievement of this threshold.

\subsection{Recommendations}

The model used assumed that the use of contraceptives can be increased from the current $46 \%$ to $70 \%$. This is only valid if intensive public health campaign is done. The simulated results depend on the effective implementation of the strategy. It is also assumed that the subjects will be willing to use the contraceptives as a control measure of population. The Kenyan population as guided by the simulated results will rise too high if no control is applied. The current fertility rate of 4.6 is too high and many strategies ranging from policy to therapeutic should be implemented. It is also recommended that the government either increase the salaries of the employees to enable every individual to support two people on average. This can be implemented through measures which reduces the rate of expenditures for example free education and free medical cover for the old and the young.

\section{References}

[1] W. C. ALLEE, Co-operation among animals, American Journal of Sociology, 37 (1931), pp. 386-398.

[2] ANTHENA MAKROGLOU, JIAXU LI and Y. KUANG, Mathematical models and software tools for the glucoseinsulin regulatory system and diabetes: an overview, Applied Numerical Mathemaics, 56 (2006), pp. 559-573.

[3] O. O. APENTENG, Demographic Modelling of Human Population Growth, LAPPEENRANTA UNIVERSITY OF TECHNOLOGY, 2009.

[4] S. -L. CHUANG, The stochastic mortality modeling and the pricing of mortality/longevity linked derivatives, 2013.

[5] P. COX, Mathematical Models for the Growth of Human Populations. By JH Pollard. [Pp. xii+ 186. Cambridge University Press, 1973. £ 5. 80.] Population Dynamics. Edited by TNE Greville. [Pp. ix+ 445. Academic Press, London, 1972. £ 6. 75.], (1973).

[6] L. EULER, A general investigation into the mortality and multiplication of the human species, Theoretical Population Biology, 1 (1970), pp. 307-314.

[7] D. GONZE, Discrete age-structured models: The Leslie matrices, (2015).

[8] L. A. GOODMAN, An elementary approach to the population projection-matrix, to the population reproductive value, and to related topics in the mathematical theory of population growth, Demography, 5 (1968), pp. 382-409.

[9] M. GUILLOT, The effect of changes in fertility on the age distribution of stable populations, Demographic Research, 20 (2009), pp. 595-598.

[10] H. HAARIO, E. SAKSMAN and J. TAMMINEN, An adaptive Metropolis algorithm, Bernoulli (2001), pp. 223-242. 
[11] D. HANSEL, G. MATO, C. MEUNIER and L. NELTNER, On numerical simulations of integrate-and-fire neural networks, Neural Computation, 10 (1998), pp. 467-483.

[12] M. KOT, Elements of mathematical ecology, Cambridge University Press, 2001.

[13] L. LEFKOVITCH, The study of population growth in organisms grouped by stages, Biometrics (1965), pp. 1-18.

[14] P. H. LESLIE, On the use of matrices in certain population mathematics, Biometrika, 33 (1945), pp. 183-212.

[15] P. H. LESLIE, Some further notes on the use of matrices in population mathematics, Biometrika, 35 (1948), pp. 213-245.

[16] T. R. MALTHUS, An Essay on the Principle of Population, as it Affects the Future Imporvement of Society, with Remarks on the Speculations of Mr. Godwin, M. Condorcet, and Other Writers, The Lawbook Exchange, Ltd., 1798.

[17] W. J. MEYER, Concepts of mathematical modeling, Courier Corporation, 2012.

[18] D. M. MORENS, G. K. FOLKERS and A. S. FAUCI, The challenge of emerging and re-emerging infectious diseases, Nature, 430 (2004), pp. 242-249.

[19] W. H. ORGANIZATION, The world health report 2000: health systems: improving performance, World Health Organization, 2000.

[20] W. E. RICKER, Computation and interpretation of biological statistics of fish populations, Bull. Fish. Res. Bd. Can., 191 (1975), pp. 1-382.

[21] F. M. SCUDO, Vito Volterra and theoretical ecology, Theoretical population biology, 2 (1971), pp. 1-23.
[22] L. SIGLER, Fibonacci's Liber Abaci: a translation into modern English of Leonardo Pisano's book of calculation, Springer Science \& Business Media, 2003.

[23] A. TSOULARIS and J. WALLACE, Analysis of logistic growth models, Mathematical biosciences, 179 (2002), pp. 2155.

[24] M. B. USHER, Developments in the Leslie matrix model, Mathematical models in ecology (1972), pp. 29-60.

[25] M. B. USHER, A matrix approach to the management of renewable resources, with special reference to selection forests, Journal of Applied Ecology (1966), pp. 355-367.

[26] P. -F. VERHULST, Notice sur la loi que la population suit dans son accroissement. correspondance mathématique et physique publiée par a, Quetelet, 10 (1838), pp. 113-121.

[27] M. WILLIAMSON, Some extensions of the use of matrices in population theory, Bulletin of Mathematical Biology, 21 (1959), pp. 13-17.

[28] J. WU and O. L. LOUCKS, From balance of nature to hierarchical patch dynamics: a paradigm shift in ecology, The Quarterly review of biology, 70 (1995), pp. 439-466.

[29] http://listcrux.com/10-effective-ways-to-control-population/ Accessed on 28th July 2016, at $12.21 \mathrm{pm}$

[30] https://www.theguardian.com/globaldevelopment/datablog/2013/dec/12/kenya-how-changedindependence-data. Accessed on 28/07/2016 at $1.30 \mathrm{pm}$

[31] http://databank.worldbank.org/data/reports.aspx?source=2\&se ries=SP. DYN. LE 00. IN \&country=\#. Accessed on $28 / 07 / 2016$ at $1.42 \mathrm{p}$. 